Ann. Génét. Sél. anim., I977, 9 (4), 41 3-422.

\title{
Corrélations entre production d'œufs et variables liées à la consommation alimentaire chez des poules rationnées en période de ponte
}

\author{
P. MÉRAT et A. BORDAS \\ Laboratoire de Génétique factorielle, \\ Centre national de Recherches zootechniques, I.N.R.A., \\ 78350 Jouy-en-Josas
}

\begin{abstract}
Résumé
Des pondeuses d'une souche Rhode-Island en cages individuelles ont été d'abord nourries " ad libitum ", leur consommation et leur production étant enregistrées, puis elles ont été rationnées à une quantité journalière fixe durant deux périodes consécutives. Les résultats étaient analysés séparément pour le groupe des poules initialement les plus lourdes et les plus légères. La ponte en restriction était en corrélation positive avec la ponte antérieure en alimentation non limitée, en corrélation négative avec le poids corporel (surtout chez les poules "lourdes"), avec le gain de poids dans la période correspondante, et avec la consommation " résiduelle "d'aliment en période non restreinte (surtout chez les poules "légères"). Ces résultats préliminaires sont brièvement discutés dans l'optique d'une sélection possible pour la ponte en présence d'un rationnement de l'aliment.
\end{abstract}

\section{Introduction}

Ces dernières annêes, de nombreuses recherches ont été faites concernant les effets d'un rationnement de l'aliment en période de ponte, suivant diverses modalités, sur les performances et le rendement économique des animaux. BraIR (I972), CAIET (I972), Summers (I972), Bougon (I974) et d'autres ont fait le point des travaux sur cette question.

Les résultats ne sont pas toujours concordants quant aux effets d'un tel traitement sur la ponte, le poids moyen des œufs et l'économie de la production. Par exemple, selon Walter et Ait ken (I96r) ou Proudfoot et Lamoreux (I973), une réduction d'environ ro $p$. cent de la quantité d'aliment consommée diminue la ponte, le poids total des œufs et ne procure pas de bénéfice. Par contre, BougoN (I974), avec un rationnement de 6 p. Ioo, BELL (I974), GERRY et MUIR (I976), trouvent une efficacité améliorée. Les résultats peuvent dépendre non seulement de l'importance de la restriction, mais de ses modalités. Ainsi, Simon (r973) obtient de 
bons résultats en limitant le temps des repas, mais fait observer qu'il s'agissait de poules entraînées depuis le jeune âge à consommer leur ration journalière en 2 à $3 \mathrm{~h}$.

En dehors d'un contraste entre souches légères et souches lourdes, il semble qu'on ait rarement recherché des différences d'ordre génétique dans la réponse à un rationnement pendant la ponte. LECLERCQ et al. (I975) mentionnent une différence à cet égard entre deux croisements commerciaux de type " ponte " et de poids corporel comparable, l'un tendant à privilégier davantage le besoin d'entretien par rapport à la production dans ces conditions.

Nos présents résultats, de caractère très limité et préliminaire, ne représentent pas une réponse à cette question. Par l'examen de corrélations entre la ponte en restriction et des variables associées à la consommation et à l'utilisation de l'aliment en registrées en même temps ou avant la restriction, ils apportent cependant quelques indications quant au résultat possible d'une sélection sur les performances de ponte en présence d'un rationnement.

\section{Matériel et Méthodes}

Au total 44 poules écloses en février I973 ont été placées en cages individuelles avant l'entrée en ponte. Elles faisaient partie d'une population de race RhodeIsland élevée à Jouy-en-Josas, issue d'une souche sélectionnée à l'origine à la Station Expérimentale d'aviculture du Magneraud. A partir de 8 mois d'âge, toutes les poules étant entrées en ponte, la consommation individuelle d'alimentétait contrôlée pendant $28 \mathrm{j}$ en novembre 1973 avec distribution ad libitum; pendant deux périodes ultérieures consécutives de 28 jours également (la première débutant en février I974), une quantité limitée d'aliment était distribuée individuellement chaque jour.

L'aliment, donné sous forme de farine, contenait I6 p. cent de protéines et $2520 \mathrm{Kcal} / \mathrm{kg}$ d'énergie métabolisable.

Pour la détermination de la restriction, les poules étaient divisées en deux groupes selon leur poids corporel en début d'expérience, un groupe "léger " de poids inférieur à $2050 \mathrm{~g}$, et un groupe "lourd" de poids supérieur à cette valeur, voisine de la moyenne générale de la population. Pour chaque groupe, à la quantité moyenne d'aliment consommée pendant la périođe "ad libitum " était retranchée une quantité calculée à partir de l'équation de régression multiple (BYERLY, I94I) :

$$
\mathrm{A} 1=a \mathrm{P} \alpha+b \Delta \mathrm{P}+\mathrm{CE}
$$

permettant de prévoir la quantité d'aliment consommée par $28 \mathrm{j}$ à partir du poids moyen $\mathrm{P}$, de la variation de poids $\Delta \mathrm{P}$ et du poids total d'œufs pondus Edurant cette période. La consommation moyenne " ad libitum " était de $3232 \mathrm{~g}$ (soit II $5,4 \mathrm{~g} / \mathrm{j}$ ) pour les poules "légères " et $3402 \mathrm{~g}$ (soit I2I,5 g/j) pour les poules "lourdes ", et la quantité retranchée (égale à $b \Delta \mathrm{P}+0, \mathrm{I} \mathrm{cE}$ ) représentait approximativement dans les deux cas I2 p. cent de l'aliment ingéré " ad libitum ». En fin de compte, durant les deux mois de restriction, chaque poule "légère " recevait par jour roI,4 $\mathrm{g}$ d'aliment et chaque poule "lourde " Io6,8 $\mathrm{g}$.

Les 44 poules étudiées étaient issues de pères différents, mais l'effectif total n'a pas été jugé suffisant pour rechercher une variation familiale de la réponse au rationnement. Nous présentons ici uniquement, outre l'effet moyen dela restriction (test $t$ ), les corrélations phénotypiques entre plusieurs variables caractérisant la production 


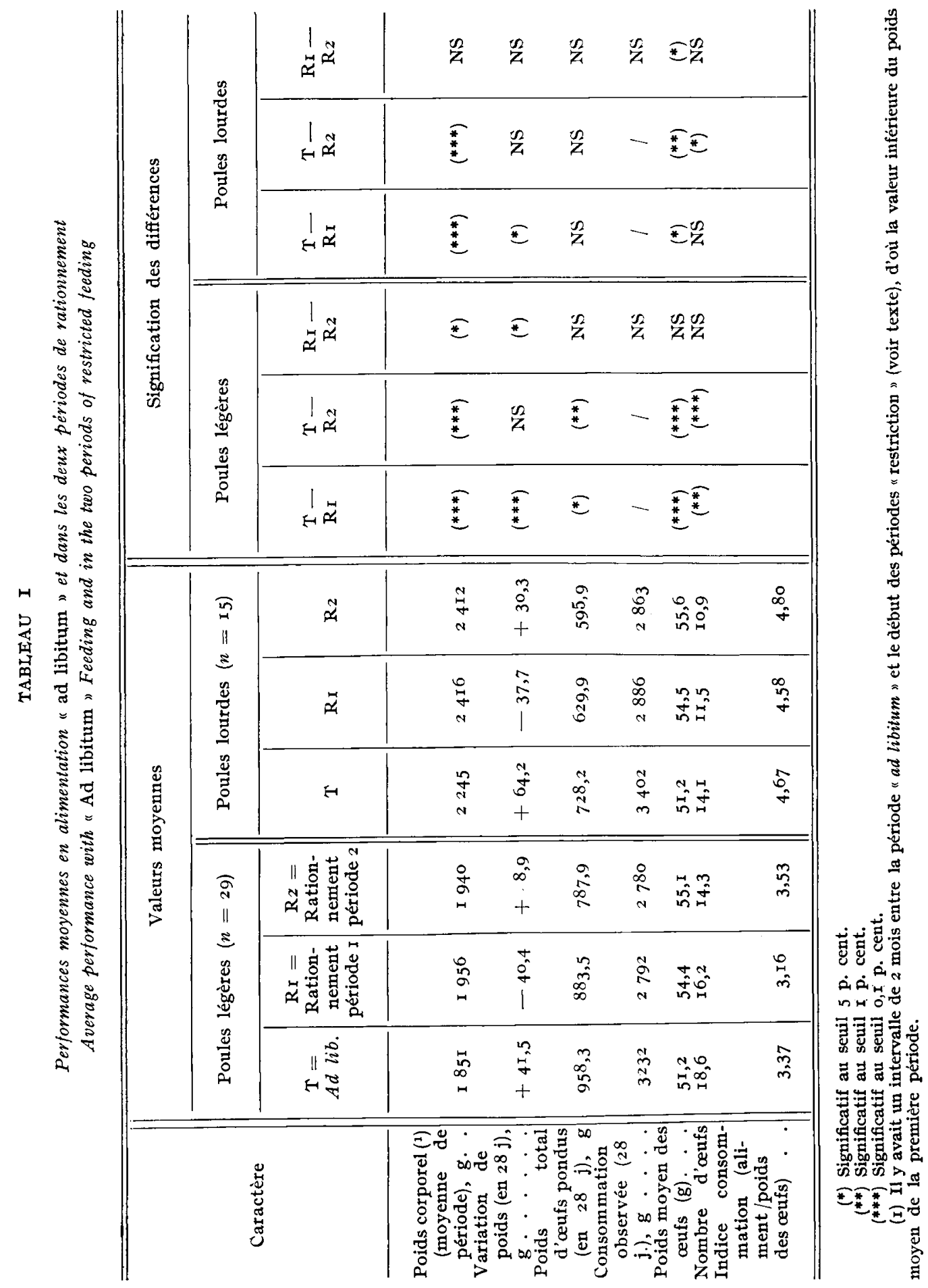


P. MÉRAT, A. BORDAS

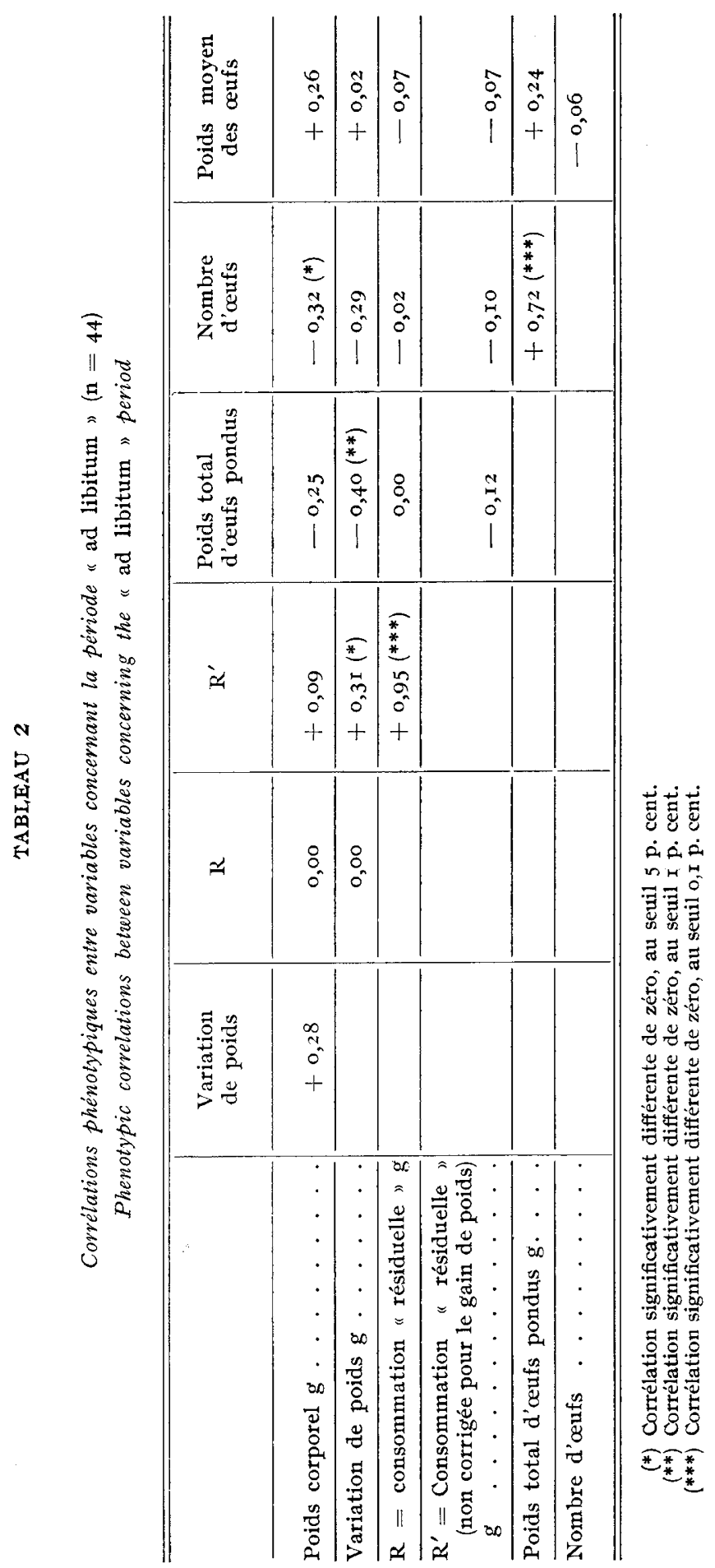




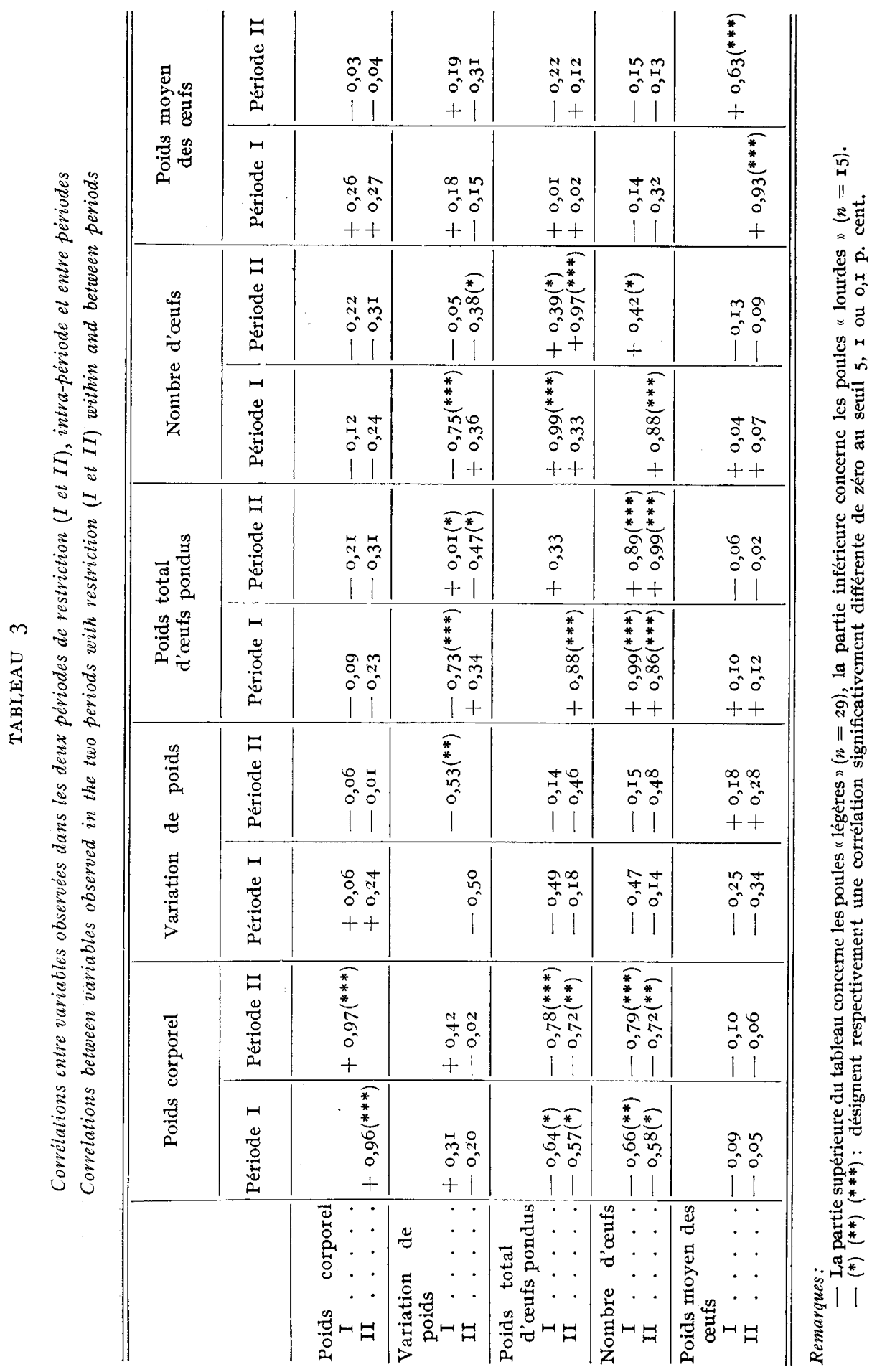




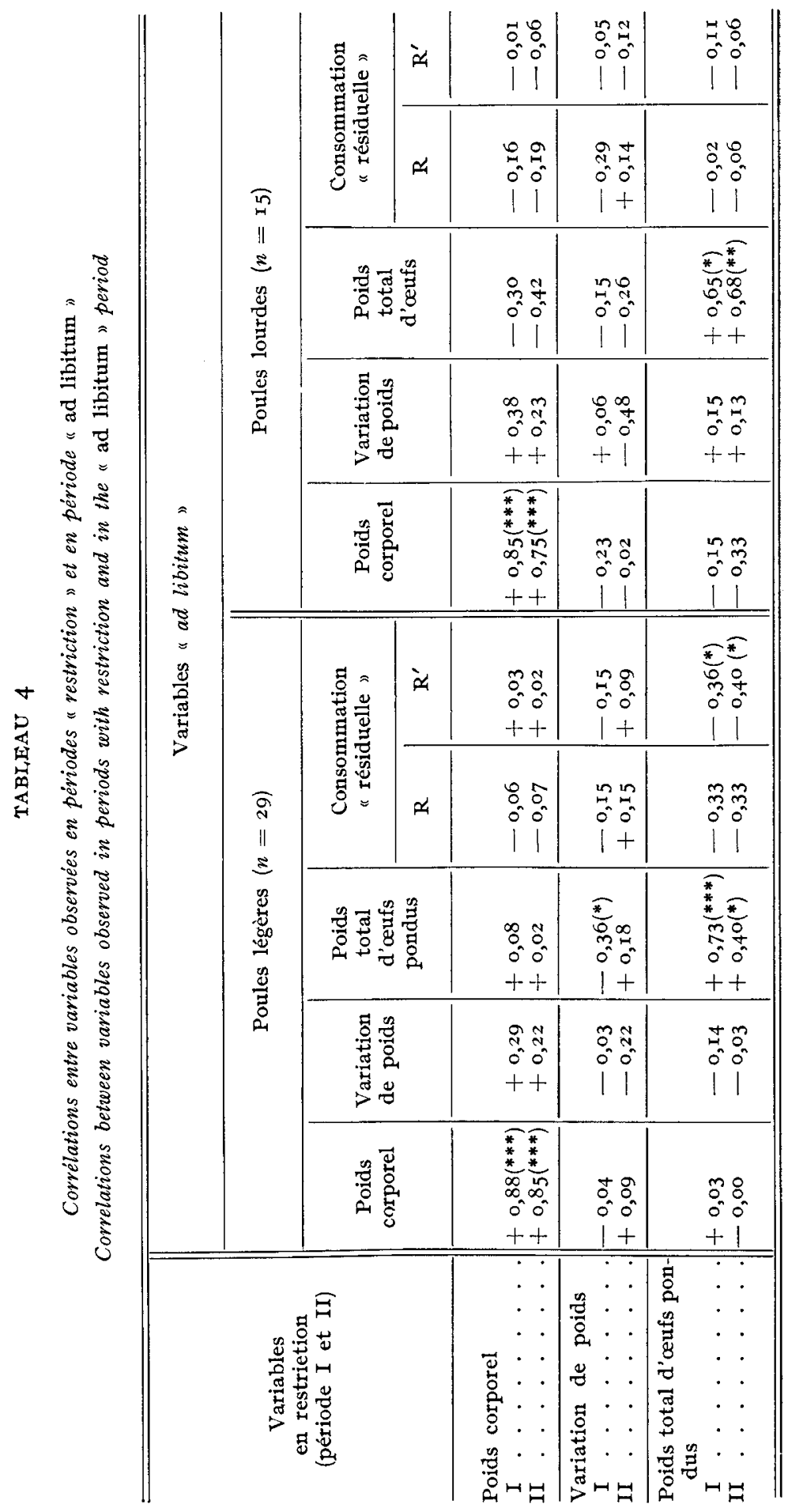


d'œufs, la consommation et le rendement alimentaire. Ces variables sont le poids moyen $\mathrm{P}$ dans chaque période, la variation de poids $\Delta \mathrm{P}$ et le poids total d'œufs pondus $\mathrm{E}$ pendant cette période. Dans certains cas sont considérés également le nombre d'œufs et le poids moyen des œufs par période. Ennfin, dans la période " ad libitum " sont calculées par individu la consommation " résiduelle " $\mathrm{R}$ (définie comme la différence entre la consommation observée et son estimation à partir de l'équation de régression mentionnée plus haut) et la variable $R^{\prime}$, différence entre la consommation observée et son estimation à partir d'une équation analogue mais n'incluant pas $\Delta \mathrm{P}$.

Les corrélations sont présentées d'une part entre les variables concernant une même période (variables "ad libitum " entre elles; variables de chaque période de restriction entre elles), d'autre part entre les variables de chaque période de restriction et celles de la période " ad libitum ". Lorsqu'interviennent les variables d'une période de restriction, les corrélations correspondantes sont calculées séparé ment à l'intérieur de chaque groupe de poids (poules "légères " ou "lourdes"). Nous avons étudié séparément chaque période de restriction, malgré l'identité du traitement reçu, car la variation moyenne de poids corporel y était différente, et la production d'œufs allait en diminuant de la première à la seconde. Les corrélations les plus significatives sont ensuite estimées également en réunissant les deux périodes.

\section{Résultats}

Les performances moyennes dans les 3 périodes considérées (ad libitum et 2 périodes de rationnement) sont présentées dans le tableau I, pour chaque groupe (poules légères et lourdes).

Le tableau 2 donne les corrélations phénotypiques entre les variables étudiées en période " ad libitum »; le tableau 3 contient les corrélations des variables observées en "restriction ", dans une même période et entre les deux périodes. La partie supérieure du tableau correspond aux poules "légères ", la partie inférieure aux poules " lourdes". Dans le tableau 4 sont indiquées les corrélations entre variables enregistrées dans la période " ad libitum " et variables enregistrées pendant les 2 périodes de rationnement.

TABLEAU 5

Corrélations entre variables observées en "restriction" en groupant les deux périodes Correlations between variables observed in the "restricted" periods, these two periods being grouped

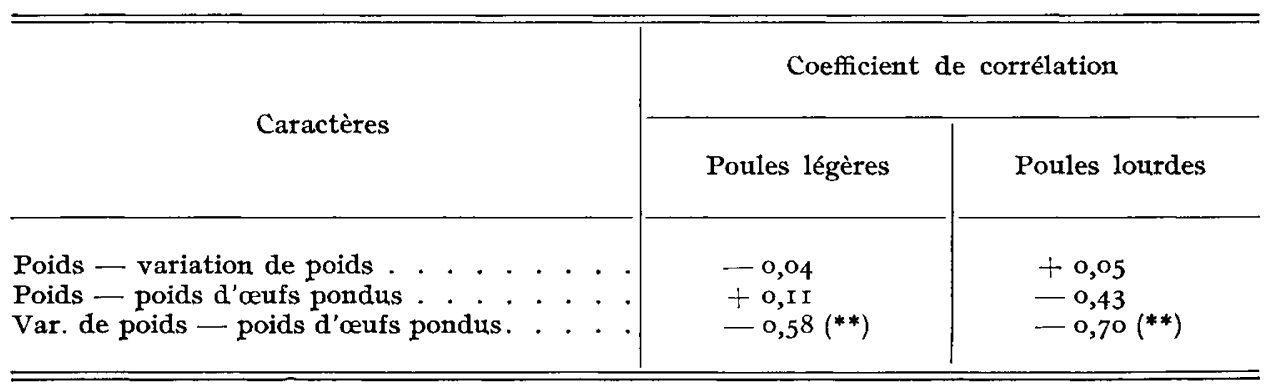


Le tableau 5 contient enfin les corrélations entre poids, variation de poids et poids d'œufs pondus en restriction, les deux périodes de restriction étant groupées en une seule.

\section{Discussion et conclusions}

Le rationnement imposé a entraîné, pendant la première période de $28 \mathrm{j}$, une baisse de poids, mais cette baisse ne s'est pas poursuivie et a même été compensée en partie pendant la période suivante. Le poids moyen des œufs, par ailleurs, a continué d'augmenter malgré la restriction.

Par contre, l'effet sur le nombre d'œufs et corrélativement sur le poids total d'œufs pondus paraît à première vue important : sur ce dernier caractère, la diminution, en pourcentage de la période "ad libitum ", est respectivement 7,8 et I $7,8 \mathrm{p}$. cent pendant la première et la seconde période cbez les poules légères, les valeurs correspondantes étant I3,5 et I8,2 p. cent pour les poules lourdes. Il ne s'agit cependant que d'une indication assez grossière, puisqu'un effet "âge " ou " stade de la courbe de ponte " se superpose à l'effet du traitement. Quant à l'indice de consommation (quantité ingérée d'aliment rapportée au poids d'œufs produits), il n'est pas amélioré. Cet indice est d'ailleurs élevé dans l'ensemble, le taux de ponte étant médiocre.

Le tableau 2 des corrélations entre variables " ad libitum " n'est donné qu'à titre indicatif, ces mêmes corrélations ayant été obtenues par ailleurs sur des données plus nombreuses (BORDAS et MERAT, I974, I976). La corrélation négative entre poids corporel et nombre d'œufs n'est pas confirmée sur des données plus importantes. Celles entre variation de poids et $R^{\prime}$, entre $R$ et $R^{\prime}$, entre nombre d'œufs et poids total d'œufs pondus, sont obligatoires et n'appellent pas d'autres commentaires. La corrélation négative entre gain de poids et poids d'œufs pondus est confirmée par celle trouvée sur un échantillon plus important (Bordas et MERAT, I976).

Plusieurs renseignements intéressants sont apportés par le tableau 3. La variation de poids dans la première période de restriction est en corrélation néga.. tive avec celle de la seconde période : Les poules perdant le plus de poids au départ en perdent moins ou en regagnent par la suite. On retrouve d'autre part, comme en régime ad libitum mais de façon apparemment plus accentuée, une corrélation négative entre gain de poids et poids d'œufs ou nombre d'œufs produits au même moment, quelle que soit la classe de poids des poules, et ceci apparaît aussi au tableau 5: La quantité d'aliment ingérée étant limitée, on peut interpréter ceci par un effet de compétition dans son utilisation, soit pour la formation d'œufs, soit pour l'engraissement. Cette corrélation n'existe qu'au niveau du nombre d'œufs et non de leur poids moyen, ce qui pourrait être rapproché des observations de LECLERCQ et al. (I975).

Une autre corrélation concerne le poids corporel et la ponte (poids total d'œufs, nombre d'œufs) : Elle est dans tous les cas négative, quoiqu'apparemment plus accentuée chez les poules lourdes. Le tableau 5 indique la même tendance en groupant les deux périodes de restriction. On peut encore penser, dans un contexte de rationnement quantitatif, à un antagonisme entre besoins d'entretien, liés au poids, et quantité de nutriments disponibles pour la ponte, les poules plus légères dans chaque groupe, avec un moindre besoin d'entretien, étant avantagées pour la production d'œufs.

Quant aux corrélations entre variables des périodes " restriction " et celles de la période " ad libitum " (tabl. 4), paraissent intéressantes celles entre poids 
total d'œufs pondus en "restriction " et d'une part la même variable en régime "ad libitum ", d'autre part la consommation résiduelle $\mathbf{R}$ ou $\mathbf{R}^{\prime}$ (également en condition " ad libitum "). La production d'œufs en restriction est en corrélation positive relativement forte, mais cependant assez éloignée de l'unité, avec celle en alimentation "ad libitum ". Par contre, elle est en corrélation négative avec la consommation "résiduelle " (surtout $R^{\prime}$ incluant le facteur "variation de poids ") de la poule non restreinte. Cette dernière corrélation n'est significative que chez les poules "légères "; pour les "lourdes ", elle est cependant de même signe. On vérifie que sa valeur reste du même ordre en groupant les deux périodes de restriction en une seule. Ici encore, il apparaît que les poules plus " économes " d'aliment pour un besoin autre que celui de production supportent mieux la restriction.

Les corrélations les plus intéressantes paraissent toutes aller dans le même sens; elles indiquent que les poules supportant le mieux la restriction imposée sont celles qui ont la dépense alimentaire la plus faible, ou le rendement le meilleur, pour leur propre entretien et pour leur gain de poids. Dans la mesure où ces corrélations, observées au niveau phénotypique, existent également au niveau génétique, elles suggèrent la possibilité d'un " pré-choix " des poules les plus aptes à produire de façon satisfaisante en milieu " rationné " à partir des performances en milieu "normal ". Ces poules seraient non seulement les meilleures pondeuses en 1'absence de rationnement, mais aussi celles ayant la plus petite taille et la meilleure efficacité alimentaire. Inversement, on peut penser à la possibilité d'une sélection indirecte sur l'efficacité alimentaire à poids corporel et ponte égaux en condition normale (ad libitum) par l'observation des performances de ponte en restriction. Des données plus nombreuses et l'examen de la réponse liée à une sélection sur ce dernier critère seront bien entendu nécessaires pour s'en assurer.

Reçu pour publication en décembre 1977.

\section{Summary}

Correlation between egg production and variables associated with feed consumption among feed-restricted hens during the laying period

Laying hens from a Rhode-Island strain in individual cages were first fed ad libitum, their feed intake and production being observed, then they were restricted to a fixed daily amount of feed during two successive periods. The results were analyzed separately for the group of hens initially heavier vs. lighter. Egg production with restricted feeding was positively correlated with production in unrestricted condition, and negatively with body weight (especially for " heavier " hens), with weight gain in the corresponding period, and with "residual "feed consumption in the $a d l i b$. period (especially for "lighter " hens). These preliminary results are briefly discussed in view of possible breeding for egg laying in presence of restricted feeding.

\section{Références bibliographiques}

BEI, D., I974. Intermittent feeding and lighting of layers. Proc. I974 Australasiam Poultry Science convention, I-IO.

BLAIR R., I972. Feed restriction in breeding birds. Feedstuffs, 44, 36-37.

Bordas A., MERat P., I974. Variabilité génétique et corrélations phénotypiques caractérisant la consommation alimentaire des poules pondeuses après correction pour le poids corporel et la ponte. Ann. Génét. Sél. anim., 6, 369-379.

bordas R., Merat P., 1976. Gain de poids pendant la ponte et sélection sur l'efficacité alimentaire chez la poule. Ann. Génét. Sél. anim., 8, 527-535. 
Bougon M. B., 1974. Influence du rationnement alimentaive sur le gain de poids et la production des pondeuses. C.R. I $5^{\mathrm{e}}$ Congrès Mondial d'Aviculture, I89-I9I.

CAIET C., I972. Intérêt et limites du rationnement chez les volailles. Atti delle Giornata avicole Internazionali, Vol. II, 389-402.

GERRY R. W., MUIR F. V., r976. Performance of Red-Rock sex-linked hens subjected to restricted feeding during the laying period. Poult. Sci., 55, I94I-I945.

LECLERCQ B., BOUCHARDEAU A., BLUM J. C., I975. Étude des effets du rationnement alimentaire de la poule sur ses performances de ponte et observations sur les effets d'une supplémentation par la bacitracine. Ann. Zoot., 24, 603-6r r.

Proudfoot F. G., LAMOREUX W. F., 1973. The bioeconomic effect of nutrient intake restriction during the rearing period and post-peak egg production feed restriction on 4 commercial meat-type parental genotypes. Poult. Sci., 52, 1269-1283.

SrmoN J., 1973. Influence d'un temps d'alimentation limité à deux heures par jour chez la poulette et la poule pondeuse de souche ponte. 4th European Poultry Conference, London, 203-210.

Summers J. D., 1972. Restricting the nutrient intake of egg production type hens. Proc. Maryland Nutrit. Confer. of feed manufacturers, 6-I4.

WALTER E. O., AITKEN J. R., I96r. Performance of laying hens subjected to restricted feeding during rearing and laying periods. Poult. Sci., 40, 345-354. 